\title{
A DISTRIBUTED MEASUREMENT SYSTEM FOR POWER QUALITY MONITORING
}

\author{
Maxim Chernyshov ${ }^{1, a}$, Maxim Nadymov ${ }^{1}$, Paul Buyankin $^{1}$, and Valery Dovgun ${ }^{1}$ \\ ${ }^{1}$ Siberian Federal University, 660041 Krasnoyarsk, Russia
}

\begin{abstract}
This paper describes a distributed power quality (PQ) monitoring system. The structure of the measurement system, format of PQ database and web-server software are being discussed. The developed software can be used for PQ analysis and research activities. Examples of harmonic pollution measurements are being displayed.
\end{abstract}

\section{Introduction}

A good power quality (PQ) is an important factor for the reliable operation of electrical loads. High penetration of nonlinear loads, such as compact fluorescent lamps, light-emitting diode (LED) lamps, switching mode power suppliers give rise to serious challenges in power quality for distribution systems. Harmonic distortion produced by nonlinear loads causes several problems, ranging from increased power losses in customer equipment, power transformers and power lines, shorter life of organic insulation to malfunction of electronic devices and telecommunication systems.

Last decade power quality issues have become serious concern for commercial and office customers. According to Electric Power Research Institute (EPRI) estimates, more than 70\% of all loads are nonlinear.

The IEEE and international standards $[1,2]$ set the limits for harmonic distortions. However they do not offer any strategy for the detection of distorting sources in the grid.

Information on the location of the distorted sources can be obtained by distributed measurement system abled to perform simultaneous measurements in different nodes of the considered grid.

This paper describes a distributed measurement system for power quality monitoring. The results of PQ measurements are reported.

\section{System Description}

The main characteristics of the measurement system are:

1. Cross-platform. The system can be accessed by all the operating systems (OS): Windows, Linux, Mac OS, etc.

2. Web browsers. The system can be accessed by different Web browsers: Explorer, Mozilla, Opera, etc.

3. Interaction. The system needs to implement the maximum level of interaction with user.

a Corresponding author : chernyshov.m.o@gmail.com 
4. Installation. The monitoring system runs without any previous installation in the client's PC.

5. Multiuser mode.

6. Distribution. Measuring devices can be installed in different network nodes.

The configuration of a power quality monitoring system is shown in Fig. 1. Its main parts are measurement units, the data acquisition system, database and Web-server.

The data acquisition system (DAS) is a server-PC that hosts the instrumentation control software and database files.

It is connected directly to the measuring units. PQ analyzers Satec PM 175 are used as measuring units.

The lab server software was developed by means of graphical language LabVIEW. LabVIEW is an industrial leader graphical programming environment for data acquisition and instrumental control [3].

The instrumentation control software is connected to the measuring units by standard RS- 485 .

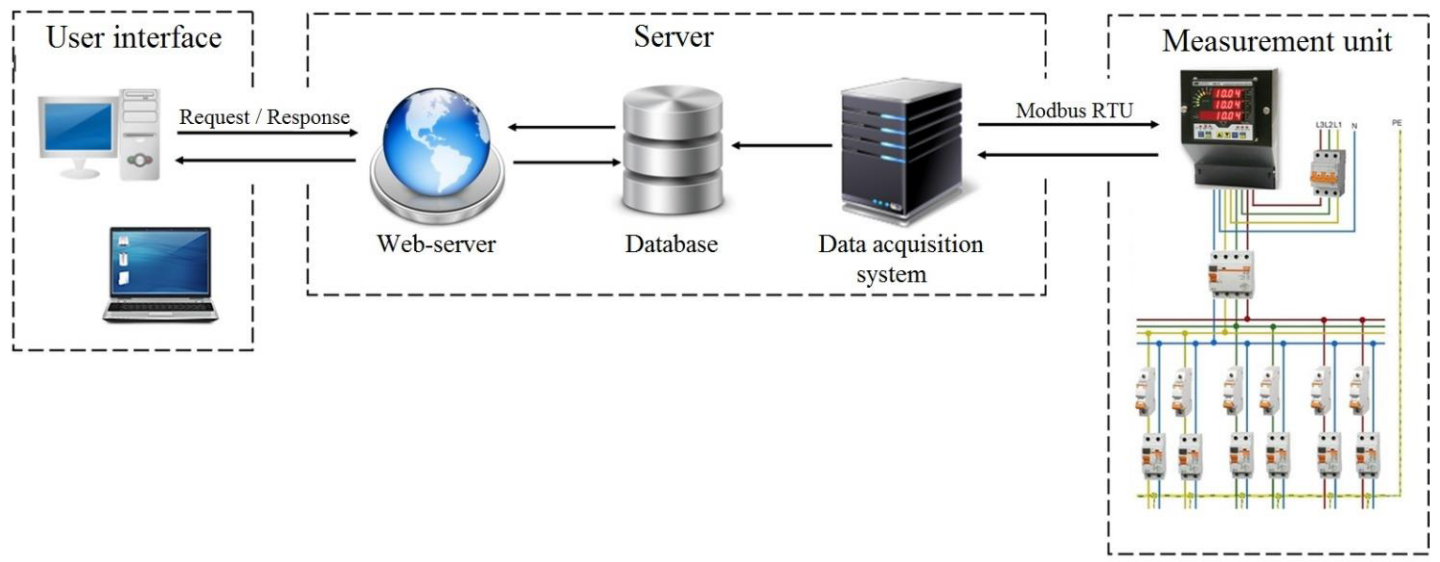

Figure 1. Configuration of a power quality monitoring system

The PQ database format is analogous to power-quality data interchange format PQDIF [4]. On a physical level, the file consists of a series of records arranged in a linked list. On a logical level file consists of a logical hierarchy of records. Such structure provides a high degree of flexibility and allows the contents to be defined logically independently of the actual physical definition.

The following quantities are measured:

The root-mean square values of voltages and currents for each phase plus neutral current.

1.The voltage and currents harmonic levels and phase angles.

2.The voltage sags and interruptions.

3.The total harmonic distortion THDU and THDI.

4.The active power.

5.The apparent power.

Different PQ indices proposed in $[5,6]$ may be evaluated by using aforementioned parameters.

\section{Web-server}

Web-server is a server-PC that hosts the web site with access to the university local network and Internet. The Apache HTTP server and the MySQL database management system were used. Apache is one of the most commonly used servers. Its main advantages are wide opportunities, ease of administration, efficiency, performance, and cross-platform.

The user interface is a web-site that runs on the user's web browser. Typically, all the computational operations are performed by the web-server. To organize the interaction of the webserver and database scripting language PHP was used. The graphical user interface is designed by means of HTML markup language and CSS, 
which are used for describing the appearance of the document. To display data from the database in the form of graphs third-party libraries developed in the JavaScript language were used. To speed up the exchange of information between client and server AJAX method was used. AJAX enables JavaScript to background data exchange between Web-server and client. It uses standard technologies that all modern Web browsers support.

Web-site allows user to visualize the power quality analyzer readings of electric energy in the form of graphs. Mechanisms of detailed setting of display schedules were implemented (setting a certain time interval, the choice of values, saving schedules in a pattern in the formats JPG, PNG, PDF). Dynamic web-site allows user to keep track of the current value and the spectral composition of the currents and voltage, THD online.

\section{Experimental Results}

The described measurement system has been implemented for PQ monitoring in power distributing system of Siberian Federal University (Krasnoyarsk, Russia). Monitoring objectives are identification of sources producing distortion and investigation of their influence on linear loads and sensitive equipment connected to the same point of common coupling. Monitoring was at the substation and selected service entrance locations.

Fig. 2 provides example of profiles for steady-state phase and neutral currents in service entrance of university building. It includes lecture rooms and computer and electrical/electronic laboratories with more than 150 computers. Nonlinear load includes fluorescent lightings, computers and airconditioners. The building is supplied by $400 \mathrm{kVA} 10 / 0,4 \mathrm{kV}$ transformer.

The neutral current had a minimum value of 10,5 A and a maximum value of $31 \mathrm{~A}$. High level of the neutral current is the result of phase imbalance and triplen harmonic currents summed in the neutral.

Fig. 3 shows harmonic distortion levels of the phase current.

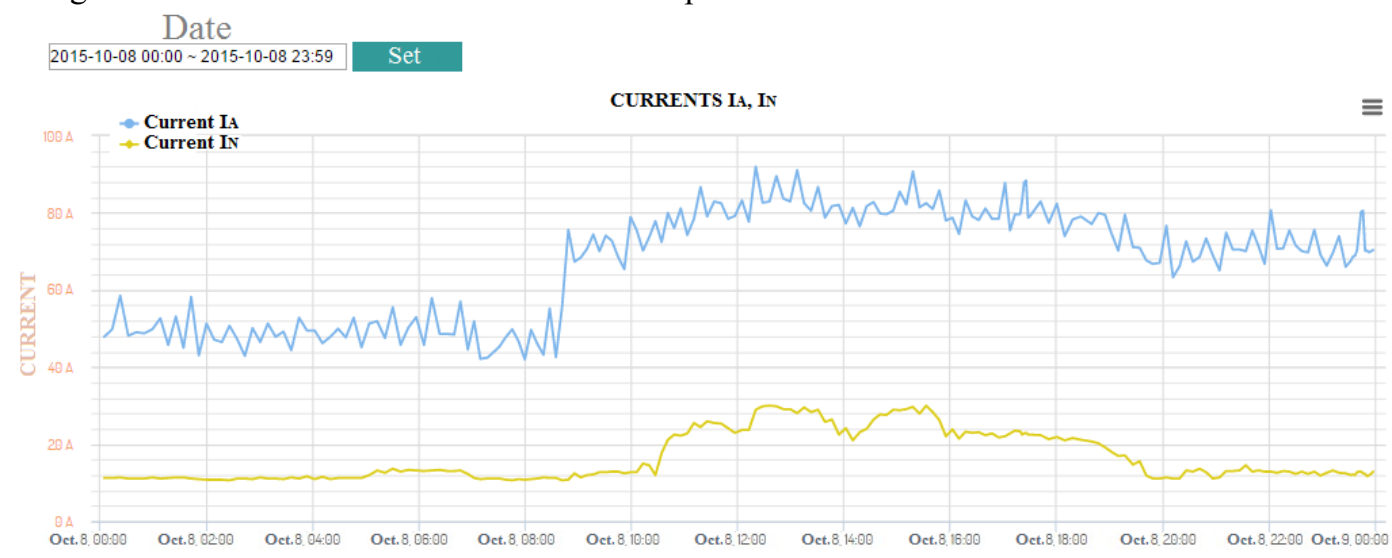

Figure 2. Phase and neutral currents 


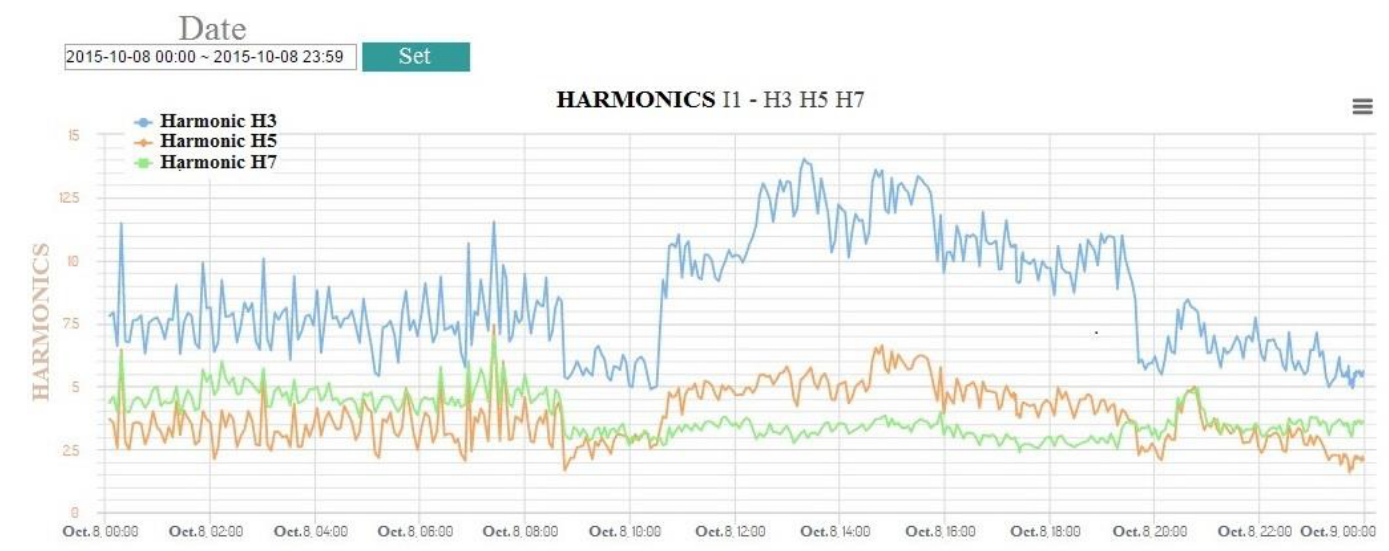

Figure 3. Harmonic distortion levels

Figs. 4 and 5 show phase current harmonic spectrum in the daytime and in the night.

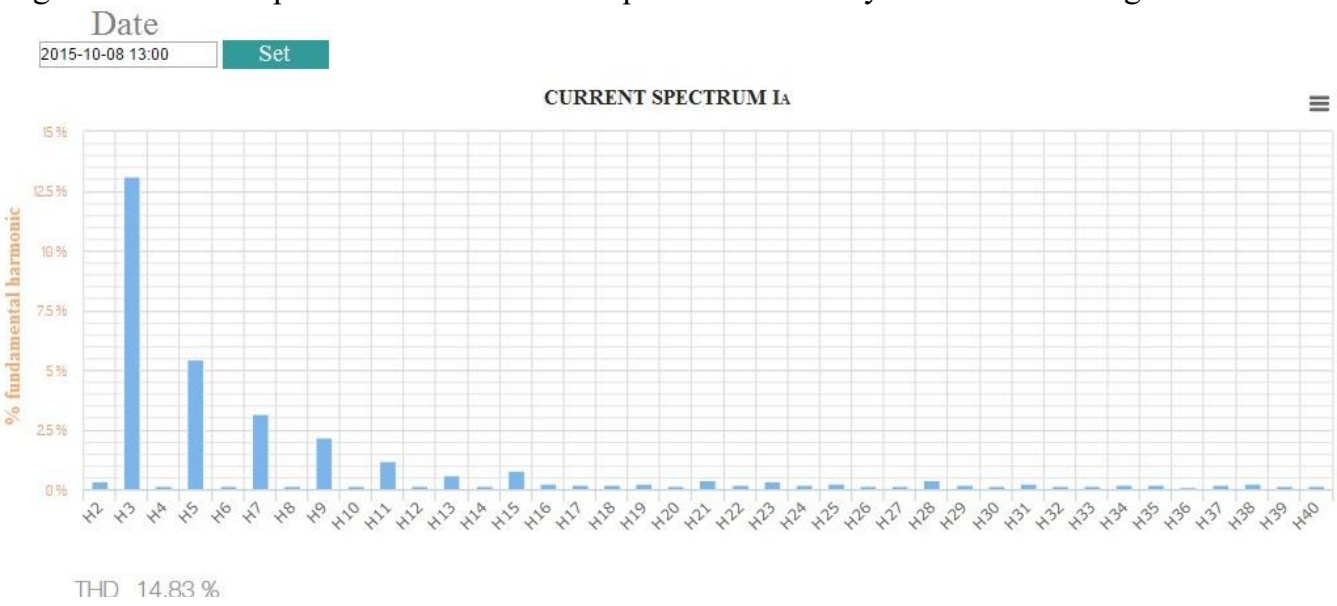

Figure 4. Current harmonic spectrum in the daytime

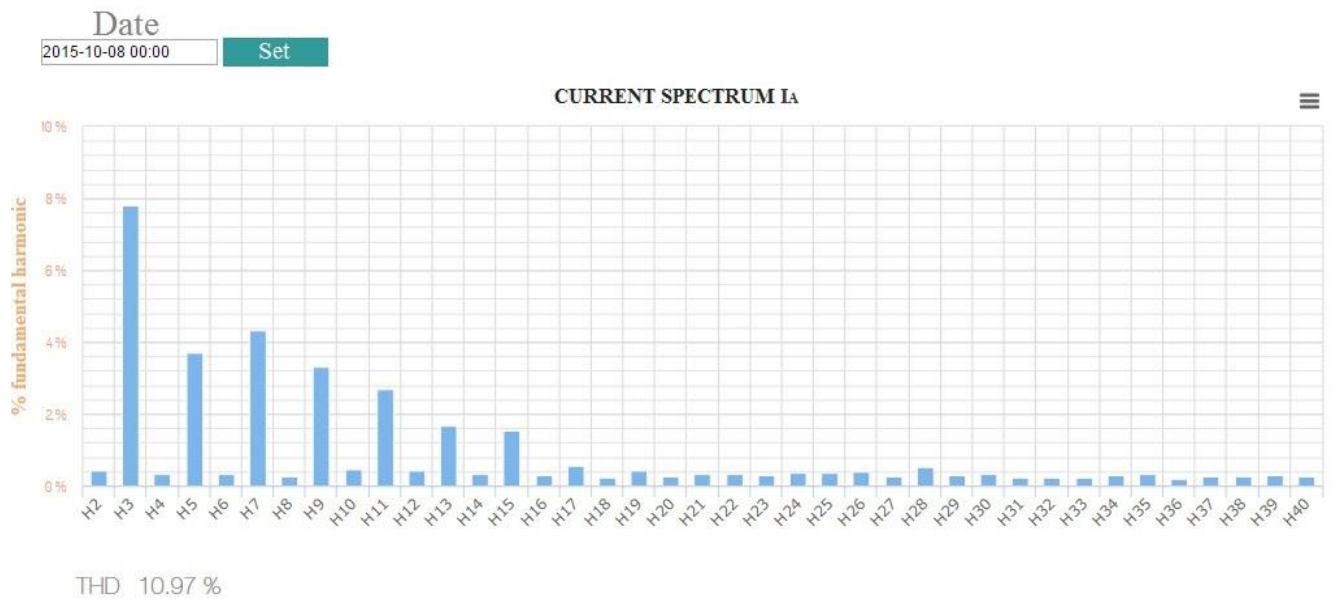

Figure 5. Current harmonic spectrum in the night 
From the results reported in Figs. 2 - 5 it can be dedused that $3^{\text {rd }}$ and $5^{\text {th }}$ harmonics are dominant. The current THD has the highest value of $14,85 \%$. According to [7] the current THD in building wiring may excess $100 \%$. This leads to higher power losses. High harmonic currents may cause overheating in the neutrals.

Future work aims at selection of optimal methods for mitigation harmonics in the university power distributing grid.

\section{Conclusion}

In this paper, a distributed measurement system for power quality monitoring was considered. The main feature of the proposed system is its capability to simultaneously estimate power quality parameters in different points of the grid. It can provide a complete information regarding harmonic state of the grid.

Proposed system can be used as a remote laboratory for research of a power grid operating with nonlinear loads.

\section{References}

1. IEEE Recommended Practices and Requirements for Harmonic Control in Electrical Power Systems (IEEE Standard, 1992)

2. IEEE Recommended Practice for Monitoring Electric Power Quality (IEEE Standard, 1995)

3. Travis J. LabVIEW for everyone. (Prentice Hall, New Jersey, 2002)

4. IEEE Recommended Practice for the Transfer of Power Quality Data (IEEE Standard, 2003)

5. Cristaldi L., Ferrero A., Salicone S. IEEE Trans. Instrum. Meas., 51 (4), 776 (2002)

6. Cataliotti A., Consentino V. IEEE Trans. Power Delivery, 25 (1), 332 (2010)

7. Lai J.-S., Key T. IEEE Trans. Ind. Appl., 33 (4), 1104 (1997) 